\title{
Dephasing effects induced by weak disorder in superlattices
}

\author{
Enrique Diez ${ }^{\mathrm{a}, \mathrm{c}, *}$, Francisco Domí nguez-Adame ${ }^{\mathrm{b}, \mathrm{c}}$, Angel Sancheż, \\ ${ }^{a}$ Departamento de Matemáticas, Escuela Politécnica Superior, Universidad Carlos III, E-28911 Leganés, Madrid, Spain \\ ${ }^{\mathrm{b}}$ Departamento de Fí sica de Materiales Facultad de Fí sicas Universidad Complutense, E-28040 Madrid, Spain \\ ${ }^{\circ}$ Grupo Interdisciplinar de Sistemas Complicados, Escuela Politécnica Superior, Universidad Carlos III, \\ E-28911 Leganés, Madrid, Spain
}

\begin{abstract}
We investigate the dephasing dynamics of Bloch oscillations in semiconductor superlattices by means of a very simple model including weak disorder and applied electric fields A thorough numerical study of our model allows us to claim that small, unintentional well width fluctuation can be responsible for fast dephasing of Bloch oscillations at low temperatures. We show that the lifetime of Bloch oscillations is controlled by a characteristic time which depends on the degree of disorder and is independent of the electric field This result is further supported by the excellent agreement between our model calculations and several recent experiments, and leads to specifi new predictions.
\end{abstract}

Keywords: Superlattices; Bloch oscillations; Disorder; Dephasing mechanisms

Dynamical effects in quantum-well semiconductors superlattices (SL's) are the basis for designing ultra-high speed electronic devices, as have been recently proposed [1]. This idea of semiconductor SL's operating at terahertz frequencies was already suggested a long time ago by Esaki and Tsu [2], who argued that electrons should undergo periodic Bloch oscillations (BO's) [3,4]: Under an applied electric fiel $F$, electrons oscillate in real space as well as in $k$ space with a characteristic period given by $\tau_{\mathrm{B}}=2 \pi \hbar / e F d, d$ being the spatial period of the SL [5-7]. The amplitude of BO's in real space is $A=\Delta / 2 e F$, where $\Delta$ is the minibandwidth. The coherent carrier motion is thus restricted to a region of length $2 A$. This periodic motion persists until the Bloch electron loses energy gained from the fiel through scattering processes. Reports of unambiguous experimental evidences for BO's in GaAs$\mathrm{Ga}_{1-x} \mathrm{Al}_{x} \mathrm{As}$ are presently available [8-12].

Inelastic scattering by phonons, deviations from SL's perfect periodicity due to unintentional imperfections, intraband scattering, interminiband transitions, and scattering by impurities severely reduce the quantum coherence required for the observation of BO's. Indeed, the scattering time $\tau$ must be larger than the Bloch period $\tau_{\mathrm{B}}$ and therefore the electric fiel must be larger than certain critical electric fiel $F_{\mathrm{c}}$ [11]. However, even in the most favorable experimental conditions $\tau$ is not much

*Corresponding author. Fax: +34 9162494 30; e-mail: diez@math.uc3m.es 
larger than $\tau_{\mathrm{B}}$ and thus only a few BO's are usually observed. The origin of such loss of quantum coherence in actual devices is far from understood and, at present, there is much debate about the role played by different scattering mechanisms in those processes. In this regard, Plessen et al. [11] found that quantum coherence is lost after few BO's in $30 \mathrm{GaAs} / 30 \mathrm{Ga}_{0.7} \mathrm{Al}_{0.3} \mathrm{As} \mathrm{SL}$ 's, which was attributed to scattering by LO phonons. On the other hand, theoretical studies point out that under most experimental conditions interminiband transitions are negligible and, consequently, cannot be responsible for the signal decay Bouchard [1]. Furthermore, Plessen et al. [11] conclude from their experimental results that the critical electric fiel $F_{\mathrm{c}}$ is higher for SL's with $\Delta$ larger than the energy of LO phonons, $E_{\mathrm{LO}}=36 \mathrm{meV}$. They explain this dependence by assuming that $\mathrm{LO}$ phonon emission is excluded when $\Delta<E_{\mathrm{LO}}$. On the contrary, Leisching et al. [12] detected up to six BO's but they did not observe any sign of a phonon threshold in SL's with $\Delta$ ranging $13-46 \mathrm{meV}$. These authors argued that the reduced sample quality of Ref. [11] could be the responsible for the threshold.

From the above discussions, it becomes clear that understanding the interplay between the electric fiel and the imperfections of the SL's is crucial to elucidate the discrepancies among different groups, either to pinpoint its relevance or to exclude it. As far as we know, however, a complete study of the effects of interface roughness on the Bloch oscillations dynamics is currently lacking. In this letter, we introduce a theoretical model for imperfect $\mathrm{GaAs}_{-} \mathrm{Ga}_{1-x} \mathrm{Al}_{x} \mathrm{As}$ SL's that successfully accounts for the experimental results. We study the dynamical behavior of these SL's subject to a dc electric fiel by measuring the position of the centroid of the wavepacket and by means of the time dependent inverse participation ratio (IPR), to be define below. These quantities will allow us to conclude that the assumption of weak disorder is enough to explain all the available experimental data, thus firml connecting the dephasing of BO's to the quality of the sample.

Interface roughness appearing during growth in actual SL's depend critically on the growth conditions [13]. For instance, protrusions of one semiconductor into the other cause in-plane disorder and break translational invariance parallel to the layers. To describe local excess or defect of monolayers, we allow the quantum well widths to fluctuat uniformly around the nominal values; this can be seen as substituting the nominal sharp width by an average along the parallel plane of the interface imperfections. Our approximation is valid whenever the mean-free-path of electrons is much smaller than the in-plane average size of protrusions as electrons only see micro-quantum-wells with small area and uniform thickness [13]. In each micro-quantum-well presents a slightly different value of its thickness and, as a consequence, resonant coupling between electronic states of neighboring GaAs layers is decreased. Therefore, in the following we will take the width of the $n$th quantum well as $a\left(1+W \epsilon_{n}\right)$, where $W$ is a positive parameter measuring the maximum fluctuation $\epsilon_{\mathrm{n}}$ 's are distributed according to a uniform probability distribution, $P\left(\epsilon_{\mathrm{n}}\right)=1$ if $\left|\epsilon_{\mathrm{n}}\right|<1 / 2$ and zero otherwise, $a$ is the nominal quantum well width. For clarity we assume that the barrier width $b$ is the same in the whole SL, although we have checked that this assumption can be dropped without changing our conclusions.

For our present purposes, it is enough to focus on electron states close to the conduction-band edge and use the effective-mass approximation. The envelope-functions for the electron wavepacket satisfie the following quantum evolution equation

$i \hbar \frac{\partial \Psi(x, t)}{\partial t}=\left[-\frac{\hbar^{2}}{2 m^{*}} \frac{d^{2}}{d x^{2}}+V(x)-e F x\right] \Psi(x, t)$,

where $x$ is the coordinate in the growth direction and the SL potential at flatban is $V(x)=\Delta E_{\mathrm{c}}$ if $x$ lies 
inside the barriers and zero otherwise, $\Delta E_{\mathrm{c}}$ being the conduction-band offset. We have considered a constant effective-mass $m^{*}$ at the $\Gamma$ valley for simplicity, but our numerical results should qualitatively describe actual SL's with position-dependent effective-mass.

We study the quantum dynamics of an initial Gaussian wavepacket

$$
\Psi(x, 0)=\left[2 \pi \sigma^{2}\right]^{-1 / 4} \exp \left[\frac{i k_{0} x-\left(x-x_{0}\right)^{2}}{4 \sigma^{2}}\right],
$$

where the mean kinetic energy is $\langle E\rangle=\hbar^{2} k_{0}^{2} / 2 m^{*}$ and $\sigma$ measures the width of the electron wavepacket. We stress that, according to Bouchard and Luban [1] the dynamical behavior of this initial state is similar to that of more realistic functions. The solution of Eq. (1) is accurately obtained using the Cayley's form for the finit difference representation of its formal solution [14,15]. Once the solution is obtained, we evaluate the position of the centroid of the wavepacket as

$X(t)=\int_{-\infty}^{\infty} \mathrm{d} x\left(x-x_{0}\right)|\Psi(x, t)|^{2}$,

which should display BO's. Moreover, we use the time-dependent inverse participation ratio (IPR), define as the second moment of the probability density

$\operatorname{IPR}(t)=\int_{-\infty}^{\infty} \mathrm{d} x|\Psi(x, t)|^{4}$,

to evaluate the spatial extent and the degree of localization of electronic wavepackets. We note that delocalized states present small IPR while localized states have larger IPR.

We have considered the same parameters as those of the SL's used in previous experiments [11,12]. In particular, we present here results for the firs one of these SL's, i.e., 100 periods of $30 \mathrm{GaAs}$ and $30 \mathrm{Ga}_{0.7} \mathrm{Al}_{0.3} \mathrm{As}$ [11] In this case, the conduction-band offset is $\Delta E_{\mathrm{c}}=0.25 \mathrm{eV}$, and the effective mass is $m^{*}=0.067 m_{\mathrm{e}}, m_{\mathrm{e}}$ being the electron mass. Samples are labeled according to their period length $d=a+b$, namely 60 SL. Similar results are obtained with the other SL's like the 84, 97, or $128 \AA$ ( $b=17 \AA, a=67,80$ and $111 \AA$, respectively), i.e., the ones reported by Leisching et al. [12], although we do not present here these results for brevity. We have straightforwardly calculated the minibandwidth for the $60 \mathrm{SL}$ obtaining $\Delta=90 \mathrm{meV}$, being larger than $E_{\mathrm{LO}}$. We study applied electric fiel in the ranges from 5 up to $20 \mathrm{kV} / \mathrm{cm}$. The fluctuatio parameter runs from $W=0$ (perfect SL's) up to $W=0.20$ (strongly disordered SL's). This maximum value considered here represents excess or defect of a few monolayers. This value is above the degree of perfection now achievable with MBE, so that realistic results are comprised within this range and we do not need to analyze stronger disorder values.

Fig. 1 displays the centroid position of the wavepacket in the $60 \mathrm{SL}$ for $F=10 \mathrm{kV} / \mathrm{cm}$ and different values of the unintentional disorder. The initial Gaussian wavepacket is located in the centermost quantum well with $\sigma=300 \AA$ and $k_{0}=0$. In Fig. 1(a), for an ordered SL, we observe the occurrence of very well define BO's with amplitude $2 A=900 \AA$ and period $\tau_{\mathrm{B}}=0.7 \mathrm{ps}$, in excellent agreement with the theoretical predictions $2 A=900 \AA$ and $\tau_{\mathrm{B}}=0.69 \mathrm{ps}$. Notice, however, that the perfect oscillatory pattern detected in periodic SL's (upper panel) is progressively destroyed upon increasing the degree of disorder as seen in the rest of panels in Fig. 1 , for $W=0.01,0.03,0.05,0.10$, and 0.20 . It is most 


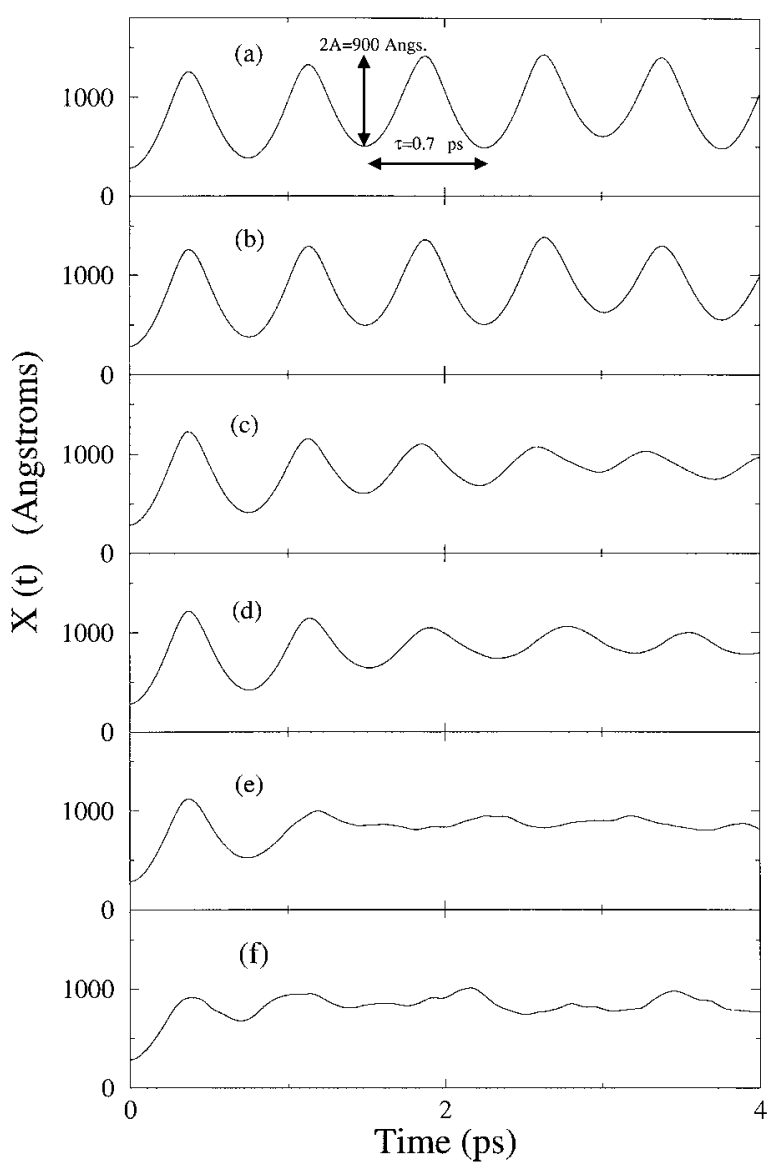

Fig. 1. Centroid of an initial Gaussian wavepacket with $k_{0}=0$ and $\sigma=300 \AA$ as a function of time in 60 SL's. The applied electric fiel is $F=10 \mathrm{kV} / \mathrm{cm}$. From top to bottom (a) $W=0$, (b) 0.01 , (c) 0.03 , (d) 0.05 , (e) 0.10 , and (f) 0.20 . The values of the amplitude $2 A=900 \AA$ and Bloch period $\tau=0.7 \mathrm{ps}$, for the perfect SL's (a), are in excellent agreement with the theoretical predictions.

important to mention here that the results do not depend on the particular realization of disorder. We note that those values correspond, if we assume that a monolayer width of this type of SL's is about 3 $\AA$, to a maximum excess of defect of less than one monolayer $(W=0.01$ and 0.03$)$, one monolayer $(W=0.05)$, two monolayers $(W=0.10)$ and four monolayers $(W=0.20)$. The disorder induces a decrease of the amplitude of the oscillations and, besides, it produces a progressive dephasing comparing with the ideal perfect case [Fig. 1(a)]. In the strong disorder case no signs of BO's are found. This fact can be explained by the absence of translational invariance at flatban and, consequently, by scattering of electrons with the random potential. Similar results are obtained with the SL's reported by Leisching et al. [12]. We have to note in this respect that for those samples we have observed a slower decay of BO's, the slowest evolution corresponding to the SL with the narrowest miniband. This can be understood by noting that the amplitude of BO's is proportional to the minibandwidth: Thus, the coherent motion of electrons takes place in a smaller region when 
decreasing the mini band-width and, therefore, carriers are less influence by disorder. This result is in very good agreement with the experimental observations [12].

We can achieve a better resolution of the BO's period and the influenc of the disorder by means of the IPR. The upper panel of Fig. 2 presents the results for the IPR of the $60 \AA$ SL when the initial Gaussian wavepacket is located in the centermost quantum-well with $\sigma=20 \AA$ and $k_{0}=0$. The electric fiel is $F=10 \mathrm{kV} / \mathrm{cm}$. In the absence of imperfections, the IPR displays a periodic pattern with marked peaks at times $t_{n}=n \tau_{\mathrm{B}}$, where $n$ is any arbitrary, nonnegative integer and $\tau_{\mathrm{B}}=0.7 \mathrm{ps}$. This means that the initial localized state is recovered after this time. It is most important, to assess the accuracy of our calculation, to mention that the numerical value of the IPR at maxima is exactly the same than that obtained from eq. (2) and eq. (4), that is, $\operatorname{IPR}(0)=1 /(2 \sqrt{\pi} \sigma)=0.01 \AA^{-1}$. Results corresponding to disordered SL's with the same initial conditions as before are shown in the remaining panels of Fig. 1, confirmin that BO's progressively disappear on increasing the degree of disorder.

From the above results we are led to the conclusion that there exists a characteristic scattering time $\tau_{\text {dis }}$ after which BO's are destroyed even by weak disorder. Moreover, it is readily observed in Fig. 2 that $\tau_{\text {dis }}$ decreases upon increasing the degree of disorder. However, the above results have been obtained for a fixe value of the electric field but clearly a meaningful definitio of the scattering time should be independent of the value of the electric field To check the validity of the introduced $\tau_{\text {dis }}$ we have studied the IPR for different values of the applied electric fiel at a given degree of disorder. Representative results are presented in Fig. 3 for $W=0.03$ (on average less than one monolayer) and $F=5,10,15$ and $20 \mathrm{kV} / \mathrm{cm}$. From this plot we can estimate that $\tau_{\text {dis }} \simeq 2.5 \mathrm{ps}$ for all

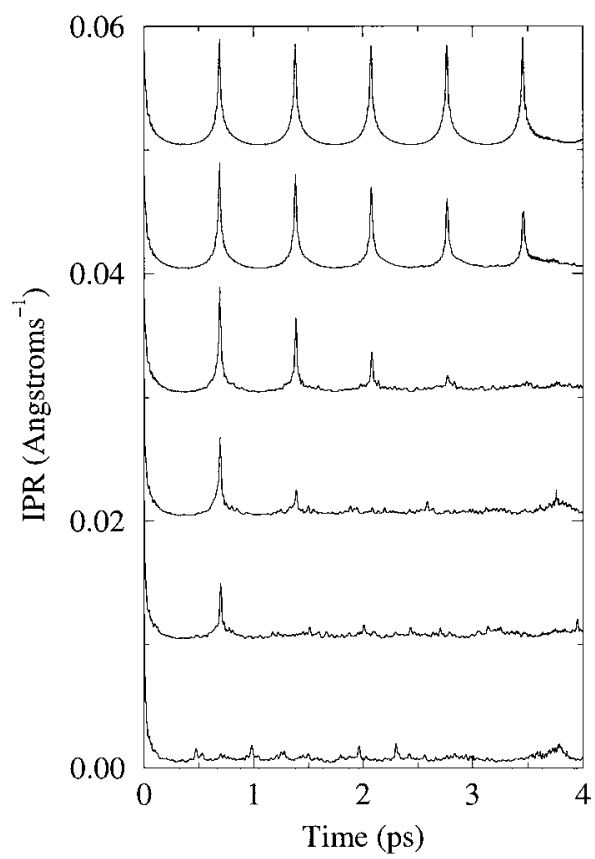

Fig. 2. IPR vs time for an initial Gaussian wavepacket with $k_{0}=0$ and $\sigma=20 \AA$, subject to an electric fiel $F=10 \mathrm{kV} / \mathrm{cm}$ in 60 SL's. From top to bottom $W=0,0.01,0.03,0.05,0.10$, and 0.20 . 


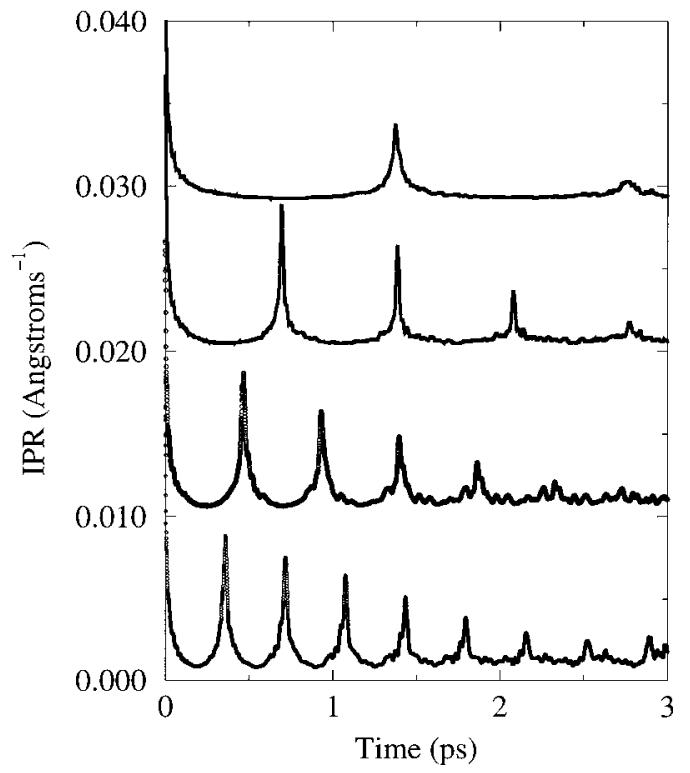

Fig. 3. IPR vs time for an initial Gaussian wavepacket with the same parameters as in Fig. 2, placed in a $60 \AA$ SL's with $W=0.03$. From top to bottom $F=5,10,15$, and $20 \mathrm{kV} / \mathrm{cm}$.

values of the electric field Thus, this scattering time plays the same role as the scattering time arising from inelastic interactions, in the sense that $\tau_{\mathrm{B}}$ must be kept smaller than $\tau_{\text {dis }}$ to observe BO's. Interestingly, this value is the same as that obtained in the experiments of Plessen et al. [11]. The scattering time increases when the mini band-width decreases, for the same amount of disorder, and values obtained with our model turn out to be perfectly consistent with all the experimental values $[9,12,16]$.

To conclude, we have been able to firml connect BO's suppression and dephasing in actual SL's to small deviations from exact flatnes at well-barrier interfaces. Specificall we have shown that an average degree of imperfection of less than a monolayer suffice to explain quantitatively the experimental results in $[11,12]$. Whereas the initially localized state is recovered after time $\tau_{\mathrm{B}}$ in the case of perfect $(W=0)$ SL's (regular behavior), any degree of disorder due to imperfections during growth leads to the disappearance of BO's after a few oscillations: The higher the degree of disorder the faster the vanishing of BO's. The very good agreement with previous experiments points out the crucial role of imperfections in the dynamics of actual SLs driven by electric fields Most importantly, we have been able to defin a characteristic scattering time $\tau_{\text {dis }}$, independent of the electric field after which BO's cannot be detected, this being a specifi prediction of our model that can be checked in experiments. In other words, for the BO's to be observed in actual SLs, the applied electric fiel must be larger than some critical electric fiel given by $e F_{\text {dis }} d=2 \pi \hbar / \tau_{\text {dis }}$. The existence of such a critical fiel is evidently very important from the viewpoint of practical applications of our results. $F_{\text {dis }}$ is directly related to the degree of disorder present in the sample and decreases upon increasing the quality of the sample, i.e., it is an excellent parameter to asses the performance of epitaxial growth techniques.

As we have seen, the main conclusion of this work is that the importance of weak disorder in the 
transport properties of SL's has to be underlined, in contrast with the general belief than the high quality of actual SL's allows to neglect disorder as a second order effect. In fact, our conclusion is of a quite broader scope, because weak disorder has been usually disregarded as a relevant factor in many other contexts, such as, e.g., studies of optical properties of semiconductors or electronic transport properties in general, to name a few. It has to be kept in mind that such an average of disorder of less than a monolayer is currently unavoidable, more so when preparing such long SL's (100 periods) as we have considered. We note, however, that high-frequency operating devices demand higher electric fields Therefore, for sufficientl high fields the region where coherent carrier motion takes place, namely $2 A=\Delta / e F$, is comparable to the SL's period $d$. In such a situation, the in-plane disorder is no longer well described by an ensemble of different quantum wells as we have proposed because the wavepacket only would see one quantum well in our model. Therefore more theoretical work is needed to investigate the role of imperfections and other dephasing mechanisms like excitonic effects [17,18] or electron-electron interactions [19], in the design of future, shorter period devices.

\section{Acknowledgements}

The authors thank with great pleasure collaborative and illuminating conversations with Fernando Agulló-Rueda, Karl Leo and Gintaras Valušis. This work has been supported by CICYT (Spain) under project MAT95-0325, and by DGES (Spain) PB96-0119.

\section{References}

[1] A.M. Bouchard, M. Luban, Phys. Rev. B47 (1993) 6815.

[2] L. Esaki, R. Tsu, IBM J. Res. Dev. 14 (1970) 61.

[3] F. Bloch, Z. Phys. 52 (1928) 555.

[4] C. Zener, Proc. R. Soc. London Ser. A145 (1934) 523.

[5] C.F. Hart, Phys. Rev. B38 (1988) 2158.

[6] M. Dignam, J.E. Sipe, J. Shah, Phys. Rev. B49(10) (1994) 502.

[7] F. Agulló-Rueda, J. Feldmann, in: H.T. Grahn (Ed.), Semiconductor Superlattices, World Scientific Singapore, 1995.

[8] K. Leo, P. Haring, F. Brüggemann, R. Schwedler, K. Köhler, Solid State Commun. 84 (1992) 943.

[9] J. Feldmann, K. Leo, J. Shah, Phys. Rev. B46 (1992) 7252.

[10] C. Waschke, H.G. Roskos, R. Schwedler, K. Leo, H. Kurtz, K. Köhler, Phys. Rev. Lett. 70 (1993) 3319.

[11] G. von Plessen, T. Meier, J. Feldmann, Phys. Rev. B49 (1994) 14058.

[12] P. Leisching, P. Haring Bolivar, W. Beck, Phys. Rev. B50 (1994) 14389.

[13] K.A. Mäder, L.-W. Wang, A. Zunger, J. Appl. Phys. 78 (1995) 6639.

[14] A.M. Bouchard, M. Luban, Phys. Rev. B52 (1995) 5105.

[15] E. Diez, A. Sánchez, F. Domí nguez-Adame, G.P. Berman, Phys. Rev. B54 (1996) 14550.

[16] T. Dekorsy, P. Leisching, K. Köhler, H. Kurz, Phys. Rev. B50 (1994) 8106.

[17] D.M. Whittaker, Europhys. Lett. 31 (1995) 55.

[18] G. Valušis, V.G. Lyssenko, D. Klatt, F. Löser, K.H. Pantke, K. Leo, in: M. Scheffler R. Zimmermann (Eds.), Proceedings of the 23rd International Conference on The Physics of Semiconductors, July 21-26 1996, World Scientific 1996, pp. 1783-1787.

[19] D. Yellin, R. Berkovits, Phys. Rev. B51 (1995) 4369. 